Sol, M.E., Groot, J.F. de, Zwinkels, M., Kruitwagen, C.L., Vershuren, O. Utrecht pediatric wheelchair mobility skills test: reliability, validity, and responsiveness in youths using a manual wheelchair. Physical Therapy: 2019, 8, 1098-1106

Postprint version

Journal website

Pubmed link

DOI
: https://academic.oup.com/ptj/advance-articleabstract/doi/10.1093/pti/pzz061/5426225?redirectedFrom=fulltext

: https://www.ncbi.nlm.nih.gov/pubmed/30939199

: 10.1093/ptj/pzz061

This is a Nivel certified Post Print, more info at nivel.nl

\title{
Utrecht Pediatric Wheelchair Mobility Skills Test: Reliability, Validity, and Responsiveness in Youths Using a Manual Wheelchair
}

\author{
Marleen E. Sol, Janke F. de Groot, Maremka Zwinkels, J.M. Anne Visser- \\ Meily, Cas L.J.J. Kruitwagen, Olaf Verschuren
}

M.E. Sol, PT, Research Group Lifestyle and Health, HU University of Applied Sciences Utrecht, Utrecht, the Netherlands; Center of Excellence for Rehabilitation Medicine, Brain Center Rudolf Magnus, University Medical Center Utrecht, Utrecht, the Netherlands; and De Hoogstraat Rehabilitation, Utrecht, the Netherlands. Address all correspondence to Mrs Sol at: marleen.sol@hu.nl.

J.F. de Groot, PT, PhD, Research Group Lifestyle and Health, HU University of Applied Sciences Utrecht; and Netherlands Institute for Healthcare Services Research (NIVEL), Utrecht, the Netherlands.

M. Zwinkels, PhD, Center of Excellence for Rehabilitation Medicine, Brain Center Rudolf Magnus, University MedicalCenter Utrecht; and De Hoogstraat Rehabilitation.

J.M.A. Visser-Meily, MD, PhD, Department of Rehabilitation, Physical Therapy Science \& Sports, Brain Center Rudolf Magnus, University Medical Center Utrecht; Center of Excellence for Rehabilitation Medicine, Brain Center Rudolf Magnus, University Medical Center Utrecht; and De Hoogstraat Rehabilitation.

C.L.J.J. Kruitwagen, MSc, Department of Biostatistics and Research Support, Julius Center, University Medical CenterUtrecht.

O. Verschuren, PT, PhD, Center of Excellence for Rehabilitation Medicine, Brain Center Rudolf Magnus, University Medical Center Utrecht; and De Hoogstraat Rehabilitation.

Background. The assessment of wheelchair mobility skills (WMS) in youths using a manual wheelchair is important. More information is needed regarding the psychometric properties of the newly developed Utrecht Pediatric Wheelchair MobilitySkills Test (UPWMST).

Objective. The purpose of this study was to evaluate the reliability, content validity, construct validity, and responsiveness of the UP-WMST 2.0 in youths using a manual wheelchair.

Design. This was a repeated-measurements, cross-sectional study. 
Sol, M.E., Groot, J.F. de, Zwinkels, M., Kruitwagen, C.L., Vershuren, O. Utrecht pediatric wheelchair mobility skills test: reliability, validity, and responsiveness in youths using a manual wheelchair. Physical Therapy: 2019, 8, 1098-1106

Methods. A total of 117 children and adolescents who use a manual wheelchair participated in this study. The UP-WMST 2.0 contains the same 15 WMS items as the original UP-WMST but has an adaptation of the scoring method. Test-retest reliability was estimated in 30 participants. Content validity was assessed through floor and ceiling effect analyses. Construct validity was assessed through hypothesis testing. Preliminary estimates of responsiveness were assessed in 23 participants who participated in a WMS training program.

Results. Test-retest reliability analysis showed weighted Cohen kappa coefficients ranging from 0.63 to 0.98 for all but 1 item. The total UP-WMST 2.0 score had an ICC of 0.97. No floor or ceiling effects were detected. Independent-sample $t$ test analysis confirmed our hypotheses regarding direction and difference in scores between age and diagnostic groups. Within-group analysis in the responsiveness study showed a positive significant change in UP-WMST 2.0 score (8.3 points).

Limitations. The small sample size used in the responsiveness study was a limitation of this study.

Conclusions. This study provided evidence of the test-retest reliability, content, and construct validity of the UP-WMST 2.0. It also provided initial evidence of the responsiveness of the UP-WMST 2.0 for measuring change in WMS in youths using a manual wheelchair.

People who use a manual wheelchair in daily life rely on their wheelchair and mobility skills to move around and overcome physical barriers, such as uneven surfaces, curbs, and slopes. More advanced wheelchair mobility skills (WMS) are associated with a higher quality of life, higher life satisfaction, and more community participation. ${ }^{1,2}$

Although WMS training can improve the skill level of adults who use a wheelchair and is an important part of clinical rehabilitation programs, ${ }^{3-7}$ only scarce evidence exists for the impact of WMS training in youths (children and adolescents). Two recent studies have reported on the importance of WMS training for youths using a manual wheelchair. ${ }^{8,9}$ In order to evaluate the effect of WMS training in youths, there is a need for a WMS outcome measure specifically developed for this population. Existing WMS outcome measures have been developed for adults who use a wheelchair, but none of these outcome measures have been validated for youths who use a manual wheelchair. It is important to validate an outcome measure again if it is applied in a new population, because certain items could be irrelevant or need adaptation. ${ }^{10}$ Therefore, we recently developed a new WMS outcomes measure, the Utrecht Pediatric Wheelchair Mobility Skills Test (UP-WMST). ${ }^{11}$ Earlier research on the development of the UP-WMST focused on content validity, through selection of WMS items from adult outcome measures and subsequently identifying relevant WMS items for youths using the clinical expertise of youth health care professionals, youths, and their parents. Afterward, the feasibility of administering the UP-WMST in youths using a manual wheelchair was assessed in a pilot study. ${ }^{11}$ Outcomes of this previous study resulted in a list of 15 items for assessing skills related to wheelchair mobility, as defined by the International Classification of Functioning, Disability and Health, ${ }^{12}$ in youths. To further develop and support clinical use of the UP-WMST, more information about its psychometric properties is needed.

Reliability refers to the extent to which scores of participants who have not changed are the same for repeated measurements over time (test-retest reliability) and to the degree to which the UP-WMST is free from measurement error. ${ }^{13}$ Content validity refers to the degree to which the content of the UPWMST is an adequate reflection of WMS in youths. ${ }^{13}$ The previous study ${ }^{11}$ focused on content validity through the development of a comprehensive list of relevant items for assessing WMS in youths. A final step in assessing content validity is to assess whether the UP-WMST includes a range of items 
Sol, M.E., Groot, J.F. de, Zwinkels, M., Kruitwagen, C.L., Vershuren, O. Utrecht pediatric wheelchair mobility skills test: reliability, validity, and responsiveness in youths using a manual wheelchair. Physical Therapy: 2019, 8, 1098-1106

which are comprehensive enough to demonstrate change, ie, without floor or ceiling effects. ${ }^{14}$ Construct validity refers to the degree to which the scores of the UP-WMST represent WMS in youths and is assessed through hypothesis testing. ${ }^{13}$ We hypothesize that age is positively associated with UPWMST score because of more experience in wheelchair mobility and/or increased strength or neuromotor development in older people who use a wheelchair. ${ }^{15,16}$ Furthermore, we expect a difference between diagnostic groups. In adults who use a manual wheelchair, the success rate of WMS differs per patient group, with people with a spinal cord injury having a higher success rate than people with a stroke or acquired brain disorder. ${ }^{17}$ In children who use a manual wheelchair, agility and anaerobic performance tests show better results in children with spina bifida (SB) than in children with cerebral palsy (CP). ${ }^{18,19}$ We therefore hypothesize that youths with SB will have a higher score on the UP-WMST than youths with CP. Responsiveness refers to the ability of the UP-WMST to detect change over time. ${ }^{13} \mathrm{~A}$ WMS outcome measure in adults has shown to be responsive after WMS training. ${ }^{6}$ For youths, data are available only from 1 small pilot study $(n=6)$ that reported on improvements in WMS after a 2- day WMS training program. ${ }^{9}$ To determine whether the UP-WMST is able to detect change over time, we hypothesize that there will be a significant improvement in outcome after a 6 -month WMS training program.

The purpose of this study was to evaluate the reliability, content validity, construct validity, and responsiveness of the UP- WMST in youths using a manual wheelchair.

\section{[H1]Methods}

\section{[H2]Sample}

This study is part of an ongoing larger study, the Let's Ride study $(n=117),{ }^{20}$ in youths. The database includes information from a reliability study $(n=30)$ and a WMS training program study $(n=97)$ (trialregister.nl registration number NTR5791). The WMS training program study itself consisted of a pilot study $(n=23)$, the data from which were used to estimate responsiveness, and an intervention study $(n=74)$. A content and construct validity study $(n=117)$ was conducted using measurements obtained prior to intervention in those who participated in the WMS training study or as the first of a pair of measurements in those who participated in the reliability study. When youths participated in more than 1 study $(n=10)$, only the first measurement of the first enrolled study was used for analysis of content and construct validity.

All participants used a bimanual wheelchair in the household or community in daily life. Children (5-11 years) and adolescents (12-18 years) were included if they were able to follow simple instructions. Informed consent was obtained fromall parents and all adolescents. To ascertain the sample was a good reflection of the total population of youths who use a manual wheelchair, we included participants with different diagnoses and youths who had various cognitive levels and received regular education or special education.

\section{[H2]UP-WMST and UP-WMST 2.0}

Table 1 shows the 15 WMS items assessed in this measurement tool, as well as the cutoff times that we used to modify the scoring method to create a modified version, the UP-WMST 2.0. All items, apart from item 10 ("holding a wheelie"), are performed at maximum speed. Item 8 ("door opening/closing"), item 13 ("platform ascending"), and item 14 ("platform descending") contain 2 subitems with different degrees of difficulty and are scored per subitem.

The UP-WMST 2.0 was created in close collaboration with pediatric physical therapists and a biostatistician. A few changes have been made to the scoring method in comparison with the original UP-WMST. To distinguish between the original version and the new version with the adapted scoring method, the new version will be called UP-WMST 2.0. Pediatric physical therapists collaborating in our 
Sol, M.E., Groot, J.F. de, Zwinkels, M., Kruitwagen, C.L., Vershuren, O. Utrecht pediatric wheelchair mobility skills test: reliability, validity, and responsiveness in youths using a manual wheelchair. Physical Therapy: 2019, 8, 1098-1106

research reported difficulties with timing the item "holding a wheelie" in participants who were unable to controla wheelie position. The duration of holding a wheelie was too short to time with a stopwatch. Therefore, we developed a different scoring method: a score of 1 is awarded if the participant can pop the front casters off the ground, a score of 2 is awarded if the participant is able to maintain a wheelie for more than 2 seconds, and a score of 3 is awarded if the participant is able to maintain a wheelie for more than 30 seconds.

At the same time, the original time scoring method of the UP-WMST in Sol et al ${ }^{11}$ has been converted into an ordinal scale for the UP-WMST 2.0. The use of an ordinal scale has several advantages over a time score per item or subitem. The ordinal ranks of the items and subitems can be summed up to a total score. A total score facilitates interpretation of test results and can be used to evaluate changes within or between individuals or groups. With support of a biostatistician, the time scores per item or subitem were converted into a 4-point ordinal scale per item. A rank of 0 is given when participants are unable to complete an item or the time to task completion takes more than 60 seconds.

The UP-WMST times of the 117 participants in this study were used to determine the cutoff time per item or subitem. The times of the participants who were able to complete an item within 60 seconds were used to calculate the mean times and determine the cutoff times at the 25th and 75th percentiles. Table 2 shows per item or subitem the cutoff times for the ordinal ranks. A total UP-WMST 2.0 score, with a maximum of 54 points, was calculated from the sum of the ordinal ranks per item or subitem. A higher score on the UP-WMST 2.0 indicates more advanced WMS.

\section{[H2]Procedures}

To assess test-retest reliability $(n=30)$, the UP-WMST 2.0 was administered twice within a 2-week period (median $=7$ days). Ethics approval was granted by the Medical Ethical Committee of the University Medical Center Utrecht (Utrecht, the Netherlands) for the reliability part of this study (protocol number 12-586). As the UP-WMST 2.0 is a new outcome measure, no information was available for sample size calculations. We have therefore performed sample size calculations based on the results of a similar study of adults who use a wheelchair. ${ }^{21} \mathrm{~A}$ group of 27 participants was needed to detect an ICC of $>0.80$. With a $10 \%$ dropout rate or missing data, an alpha value of 0.05 , and a power of 0.80 , a sample size of 30 participants will be required. All retests of the UP- WMST 2.0 were conducted using the same material, gym floor, tire pressure, test administrator and instructions as the first administration of the UP-WMST 2.0. The administrator explained every item verbally and when necessary showed the execution of the item by walking (eg, figure-of-eight shape). Participants could use 1 trial attempt and subsequently a maximum of 3 attempts were allowed. The best time was used and converted to a rankscore.

To assess content and construct validity $(n=117)$, the descriptive statistics of the baseline UP-WMST 2.0 scores of the total sample were used. The UP-WMST 2.0 was administered according to the protocol by a physical therapist, occupational therapist, or research team member. All administrators received a 4-hour training (theory and practice) on the administration of the UP-WMST 2.0. The Institutional Review Board of the University Medical Center Utrecht approved the study protocol for the Let's Ride intervention study (protocol number 15-136).

A subsample of participants $\left(n=23\right.$ ) participated in the Let's Ride pilot study. ${ }^{22}$ The results of this pilot study were used to estimate preliminary evidence of responsiveness. Participants followed a WMS training program aimed at propelling efficiently, going up and down curbs, holding a wheelie and negotiating the physical environment outdoors. This program was organized by a Dutch patient organization and consisted of 4 training sessions of 2.5 hours each over a 6-month period. A manual wheelchair user with more than 10 years of experience in teaching WMS conducted the training sessions. After each training session, participants were instructed and motivated to practice at home 
Sol, M.E., Groot, J.F. de, Zwinkels, M., Kruitwagen, C.L., Vershuren, O. Utrecht pediatric wheelchair mobility skills test: reliability, validity, and responsiveness in youths using a manual wheelchair. Physical Therapy: 2019, 8, 1098-1106

and received an individual video instruction about how to practice their skills. The research team administered the UP-WMST 2.0 before and after the training period using the same material, gym floor, tire pressure, and instructions.

\section{[H2]Data Analysis}

\section{[H3]Reliability.}

The test-retest reliability of the individual items or subitems was assessed using a quadratic weighted Cohen kappa coefficient. In addition, the test-retest reliability of the total score was calculated using a 2-way mixed ICC (ICC agreement). ${ }^{10} \mathrm{~A}$ priori, a weighted Cohen kappa coefficient of 0.61 or higher and an ${ }{ } C_{\text {agreement }}$ of 0.80 or higher were defined as indications of good reliability. ${ }^{23}$ The standard error of measurement (SEMagreement) and the smallest detectable change (SDC) were calculated for the total UP-WMST 2.0 score. ${ }^{10}$ The SCD was calculated as follows: SDC $=1.96 \times \quad \times$ SEMagreement.

\section{[H3]Content validity.}

Floor and ceiling effects of the total UP-WMST 2.0 score were determined as part of content validity. Floor and ceiling effects were considered present if $15 \%$ or more of the participants scored a lowest or highest possiblescore. ${ }^{10}$

\section{[H3]Construct validity.}

The construct validity of the UP-WMST 2.0 was evaluated using separate independent-sample $t$ tests to assess differences in mean UP-WMST 2.0 scores in groups defined by age (children versus adolescents) and diagnosis (SBversus CP).

\section{[H3]Responsiveness.}

The mean change and $95 \% \mathrm{Cl}$ of the UP-WMST 2.0 score after a WMS training program were assessed to determine responsiveness. A paired-sample $t$ test was used to assess whether there was a significant change in the mean score of the UP-WMST 2.0.

\section{[H2]Role of the Funding Source}

This study was funded by SIA Raak (ref. no. PRO-4-03), which provided funding for the Fit for the Future consortium. The funder played no role in the design, conduct, or reporting of this study.

\section{[H1]Results}

\section{[H2] Reliability}

Table 3 shows the descriptive statistics of the participants included per study. Thirty participants were included in the test-retest reliability study. One participant became ill and could not perform the retest of the UP-WMST 2.0 within the 2-week period. The scores of this participant were not used in the reliability analysis. The results for the test-retest reliability per item or subitem and total score are shown in Table 4. The weighted kappa coefficients ranged from 0.63 to 0.98 for all but 1 item, an indication of good reliability based on definitions the research team developed a priori. Item 7 ("sudden stop"), with a coefficient of 0.50 , was moderately reliable based on the definitions the research team developed a priori. Because of this moderate value of reliability, the "sudden stop" item has been removed from the UP-WMST 2.0 and will not be part of the total score. The new maximum score on the UP-WMST 2.0 is 51 points. The ICC agreement for the total UP-WMST 2.0 score was 0.97 , the SEMagreement was 1.80 points, and the SDC was 4.98 points. 
Sol, M.E., Groot, J.F. de, Zwinkels, M., Kruitwagen, C.L., Vershuren, O. Utrecht pediatric wheelchair mobility skills test: reliability, validity, and responsiveness in youths using a manual wheelchair. Physical Therapy: 2019, 8, 1098-1106

\section{[H2]Content Validity}

The figure shows the variation of UP-WMST 2.0 scores in 117 participants with a normal distribution and a range of 9 to 51 points. No floor or ceiling effect were present, as none of the participants had a minimum score and only $1.7 \%$ of the participants reached the maximum score. Fifteen percent of the participants reached a score of 40 points or higher on the UP-WMST 2.0.

\section{[H2]Construct Validity}

Table 5 shows the mean score, standard deviation and range of the UP-WMST 2.0 score per age group and per diagnosis. The UP- WMST 2.0 scores were significantly lower, 5.4 points (with $P<.01$ ), in children $(n=56)$ than in adolescents $(n=61)$. The UP- WMST 2.0 scores were significantly higher, 9.1 points (with $P<.001)$, in youths with $S B(n=31)$ than in youths with $C P(n=47)$. No confounding influence of age was detected when youths with SB were compared with youths with CP.

\section{[H2]Responsiveness}

Table 3 showed the descriptive statistics of the 23 participants who participated in a WMS training with their pretraining and posttraining UP-WMST 2.0 score. Two participants could not complete the program because of unrelated medical treatments, and 1 participant was not available for assessment after training. The results for these 3 participants were not included in the statistical analysis. The paired-sample $t$ test of 20 participants showed a significant $(P<.001)$ improvement of 8.3 points $(95 \%$ $\mathrm{Cl}=5.29-11.31)$.

\section{[H1]Discussion}

To our knowledge, this is the first study to evaluate measurement properties of a WMS outcome measure in youths using a manual wheelchair. Our results show positive estimates of reliability, content validity, and construct validity and preliminary evidence of the responsiveness of the UPWMST 2.0 for youths who use a manual wheelchair.

\section{[H2]Reliability}

A low kappa coefficient for the item "sudden stop" led to the removal of this item from the UP-WMST 2.0. In earlier research, the item "sudden stop" was found to be relevant to assess in youths using a manual wheelchair.11 The results of this study show that the current administration is not a reliable method and was therefore removed from the current version. Given the relevance though, future research should look into a different method of assessing this item. The total score of the UP-WMST 2.0 showed an excellent ICCagreement (0.97), comparable to the ICCs reported for WMS outcome measures in adults (0.90-0.99).17,24,25 In addition to the ICC, we calculated the SEMagreement and the SDC. This information is useful in clinical practice. The SDC of 4.98 points indicates that an individual improvement or decline of 5 or more points reflects a true change in the UP-WMST 2.0 score. This change in score is $9.4 \%$ of the total range of the UP-WMST 2.0 and is in line with previously reported detectable change scores (5\%-15\%) in 2 adult WMS outcome measures. 25 Thus, repeated periodically, the UP-WMST 2.0 can be used as a criterion for the effectiveness of rehabilitation treatment focusing on WMS in youths who use a manual wheelchair.

\section{[H2]Content Validity}

In addition to the evidence reported on content validity in the previous study on the development of the UP-WMST, ${ }^{11}$ this study assessed the comprehensiveness of the UP-WMST 2.0 through the analysis of floor and ceiling effects. The results show no floor or ceiling effect detectable in our sample and further supports the content validity of the measure. Even though no ceiling effect occurred in this study, $15 \%$ of our study sample scored in the upper ranges of this test (40 points or more). Youths with more advanced WMS may require a different outcome measure. ${ }^{9,11}$ 
Sol, M.E., Groot, J.F. de, Zwinkels, M., Kruitwagen, C.L., Vershuren, O. Utrecht pediatric wheelchair mobility skills test: reliability, validity, and responsiveness in youths using a manual wheelchair. Physical Therapy: 2019, 8, 1098-1106

\section{[H2] Construct Validity}

Independent $t$ test analysis confirmed our hypothesis regarding age and diagnoses. Youths with SB and adolescents scored significantly higher on the UP-WMST 2.0 than youths with CP and children, respectively. Even though the group of adolescents scored, on average, higher than the group of children, their lower and upper UP-WMST 2.0 limits did not differ much. Similarly, the range in scores between youths with SB and youths with CP did not differ much, despite the fact that youths with SB scored, on average, 9.1 points higher. Clinicians should be aware of these large variations in WMS within age groups or different diagnoses. Bloemen et al ${ }^{19}$ already mentioned possible explanations for the variation in wheelchair skill related fitness for children with SB, such as wheelchair features, physical factors, or propulsion techniques. Sawatzky et $\mathrm{al}^{26}$ found that speed and mechanical effectiveness explained $36 \%$ of the variance in wheelchair skills scores in adults and children who use wheelchairs. In adults who use a wheelchair, wheelchair weight ${ }^{17}$ and upper-extremity strength ${ }^{27}$ have already been shown to have an influence on wheelchair skill outcome measures. Future research may provide insight into how these factors contribute to WMS in youths.

\section{[H2]Responsiveness}

On group level we found an 8.3-point improvement, which is a $27 \%$ mean relative improvement in WMS on the UP-WMST 2.0 after a WMS training program. These results show a larger relative improvement compared to the $14 \%$ improvement reported by Sawatsky et al ${ }^{9}$ in 6 children following a WMS training program. These findings show the UP-WMST 2.0 to be sensitive to measure change in this group of participants over a 6-month period. On an individual level, participants showed variation in the magnitude of improvement. This variation could be due to wheelchair features or physical factors (as mentioned above), different effects of WMS training per participant, or lower sensitivity of the UP-WMST 2.0 in some children. The sample size for this portion of the study was too small to explore possible explanations for the observed variations. The small sample size also made it impossible to determine the minimal important change (MIC). The MIC is the smallest change in a score on the UP-WMST 2.0 that patients perceive as important. ${ }^{10}$ The MIC, together with the SDC, is useful for clinicians in interpreting changes in individual UP-WMST 2.0 scores. For use in clinical practice, future research with larger samples should examine the MIC and evaluate whether the UPWMST 2.0 is sensitive to measure change in a broad sample of youths who use a manual wheelchair.

\section{[H1]Strengths and Limitations}

The sample size used in this study is large compared to those used in other studies of youths who use a manual wheelchair. The inclusion of a heterogeneous group of participants who use a wheelchair leads to an increase in the clinical relevance and generalizability of the results of this study ${ }^{10}$. Moreover, to ascertain clinical relevance, a group of physical therapists, occupational therapists, and physical education teachers of the Fit for the Future consortium ${ }^{20}$ have been advising the research team through all the phases of the development of the UP-WMST 2.0. One of the limitations of this study was the use of several test administrators to administer the UP-WMST 2.0. Even though the administrators were trained to administer the test according to protocol, it was impossible to determine whether test administrators had a different influence on the participants' performance. For example, a participant might perform differently when the test is administered by a familiar therapist instead of an unknown research team member. At the same time, this too reflects daily clinical practice and increases the generalizability of the results. The interrater reliability of the UP-WMST 2.0 was not assessed in this study. Another limitation was the sample size in the responsiveness study. The sample size was small because of the setup of the Let's Ride pilot study. The results do give a good indication whether the test is responsive to measure change on group level, 
Sol, M.E., Groot, J.F. de, Zwinkels, M., Kruitwagen, C.L., Vershuren, O. Utrecht pediatric wheelchair mobility skills test: reliability, validity, and responsiveness in youths using a manual wheelchair. Physical Therapy: 2019, 8, 1098-1106

but no general statements can be made toward the responsiveness of the UP- WMST 2.0. The results of this study show the UP-WMST 2.0 can be used in further research toward wheelchair mobility in youths. Furthermore, the average age of the responsiveness group was younger than the average age of the total sample. Future research should evaluate the interrater reliability and responsiveness of the UP-WMST 2.0 in a larger and slightly older sample. Future outcomes of our ongoing Let's Ride study into the responsiveness of the UP-WMST 2.0 after a WMS training in a larger samplewill provide additional valuable information about the SDC compared to the MIC. These future outcomes combined with the results of this study will make the UP-WMST 2.0 more suitable for use on individual level in clinical practice.

\section{[H1]Conclusion}

The present study supports the reliability, content validity, and construct validity of the UP-WMST 2.0 for measuring WMS in youths who use a manual wheelchair and provides preliminary evidence suggesting responsiveness to change over a 6-month period.

Further research is needed to improve the interpretation of UP-WMST 2.0 scores for use in daily practice.

\section{Author Contributions and Acknowledgments}

Concept/idea/research design: M.E Sol, J.F. de Groot, O. Verschuren, J.M.A. Visser-Meily Writing: M.E Sol, J.F. de Groot, M. Zwinkels, O. Verschuren

Data collection: M.E. Sol, M. Zwinkels

Data analysis: M.E. Sol, J.F. de Groot, M. Zwinkels, J.M.A. Visser-Meily, C.L.J.J. Kruitwagen, O. Verschuren Project management: M.E Sol, J.F. de Groot, O. Verschuren

Fund procurement: J.F. de Groot, O. Verschuren

Consultation (including review of manuscript before submitting): J.F. de Groot, M. Zwinkels, J.M.A. Visser-Meily, C.L.J.J. Kruitwagen, O. Verschuren

This study is part of the Fit for the Future collaboration (H. Wittink, A. Dallmeijer, R. van den Berg-Emons). The authors thank all of the participating children, parents, and health care professionals for volunteering their time and sharing their experiences. The authors also thank all of the participating students who enthusiastically contributed to data collection and analysis in the Let's Ride study. Theauthors are especially grateful to the BOSK patient organization and K-J Projects for sharing their expertise and involvement in the Wheelchair Mobility Skills training program.

\section{Ethics Approval}

Ethics approval was granted by the Medical Ethical Committee of the University Medical Center Utrecht (Utrecht, the Netherlands) for the reliability part of this study (\#12-586). The Institutional Review Board of the University Medical Center Utrecht approved the study protocol for the Let's Ride intervention study (\#15-136).

\section{Funding}

This study was funded by SIA Raak (ref. no. PRO-4-03), which provided funding for the Fit for the Futureconsortium. 
Sol, M.E., Groot, J.F. de, Zwinkels, M., Kruitwagen, C.L., Vershuren, O. Utrecht pediatric wheelchair mobility skills test: reliability, validity, and responsiveness in youths using a manual wheelchair. Physical Therapy: 2019, 8, 1098-1106

\section{Disclosure}

The authors completed the ICJME Form for Disclosure of Potential Conflicts of Interest and reported no conflicts of interest.

\section{References}

1. Smith EM, Sakakibara BM, Miller WC. A review of factors influencing participation in social and community activities for wheelchair users. Disabil Rehabil Assist Technol. 2016;11:361-374.

2. Hosseini SM, Oyster ML, Kirby RL, Harrington AL, Boninger ML. Manual wheelchair skills capacity predicts quality of life and community integration in persons with spinal cord injury. Arch Phys Med Rehabil. 2012;93:2237-2243.

3. MacPhee AH, Kirby RL, Coolen AL, Smith C, MacLeod DA, Dupuis DJ. Wheelchair skills training program: a randomized clinical trial of wheelchair users undergoing initial rehabilitation. Arch Phys Med Rehabil. 2004;85:41-50.

4. Kirby RL, Mitchell D, Sabharwal S, McCranie M, Nelson AL. Manual wheelchair skills training for community-dwelling veterans with spinal cord injury: a randomized controlled trial. PLoS One. 2016;11:e0168330.

5. Ozturk A, Ucsular FD. Effectiveness of a wheelchair skills training programme for communityliving users of manual wheelchairs in turkey: a randomized controlled trial. Clin Rehabil. 2011;25:416-424.

6. Worobey LA, Kirby RL, Heinemann AW, et al. Effectiveness of group wheelchair skills training for people with spinal cord injury: a randomized controlled trial. Arch Phys Med Rehabil. 2016;97:1777-1784.e3.

7. Best KL, Routhier F, Miller WC. A description of manual wheelchair skills training: current practices in canadian rehabilitation centers. Disabil Rehabil Assist Technol. 2014;10:393-400.

8. Bloemen MA, Verschuren $\mathrm{O}$, van Mechelen $\mathrm{C}$, et al. Personal and environmental factors to consider when aiming to improve participation in physical activity in children with spina bifida: a qualitative study. BMC Neurol. 2015;15:11.

9. Sawatzky B, Rushton PW, Denison I, McDonald R. Wheelchair skills training programme for children: a pilot study. Aust Occup Ther J. 2012;59:2-9.

10. de Vet HCW, Terwee CB, Mokkink LB, Knol DL. Measurement in Medicine. New York, NY: Cambridge University Press; 2011.

11. Sol ME, Verschuren O, de Groot L, de Groot JF; Fit-For-the-Future!-consortium. Development of a wheelchair mobility skills test for children and adolescents: combining evidence with clinical expertise. BMC Pediatr. 2017;17:51.

12. World Health Organization. International Classification of Functioning, Disability and health (ICF). http://apps.who.int/classifications/icfbrowser/. Accessed January 30, 2019.

13. Mokkink LB, Terwee CB, Patrick DL, et al. The COSMIN study reached international consensus on taxonomy, terminology, and definitions of measurement properties for health-related patient-reported outcomes. J Clin Epidemiol. 2010;63:737-745.

14. Mokkink LB, Terwee CB, Knol DL, et al. The COSMIN checklist for evaluating the methodological quality of studies on measurement properties: a clarification of its content. BMC Med Res Methodol. 2010;10:22.

15. Larson JC, Mostofsky SH, Goldberg MC, Cutting LE, Denckla MB, Mahone EM. Effects of gender and age on motor exam in typically developing children. Dev Neuropsychol. 2007;32:543-562.

16. Ploegmakers JJ, Hepping AM, Geertzen JH, Bulstra SK, Stevens M. Grip strength is strongly associated with height, weight and gender in childhood: a cross sectional study of 2241 children and adolescents providing reference values. J Physiother. 2013;59:255-261.

17. Kirby RL, Dupuis DJ, Macphee AH, et al. The wheelchair skills test (version 2.4): measurement properties. Arch Phys Med Rehabil. 2004;85:794-804. 
Sol, M.E., Groot, J.F. de, Zwinkels, M., Kruitwagen, C.L., Vershuren, O. Utrecht pediatric wheelchair mobility skills test: reliability, validity, and responsiveness in youths using a manual wheelchair. Physical Therapy: 2019, 8, 1098-1106

18. Verschuren O, Zwinkels M, Obeid J, Kerkhof N, Ketelaar M, Takken T. Reliability and validity of short-term performance tests for wheelchair-using children and adolescents with cerebral palsy. Dev Med Child Neurol. 2013;55:1129-1135.

19. Bloemen MA, Takken T, Backx FJ, Vos M, Kruitwagen CL, de Groot JF. Validity and reliability of skill-related fitness tests for wheelchair-using youth with spina bifida. Arch Phys Med Rehabil. 2017;98:1097-1103.

20. Hogeschool Utrecht. Project informatie. https://husite.nl/fitforthefuture/project-informatie/. Accessed January 30, 2019.

21. Kilkens OJ, Post MW, van der Woude LH, Dallmeijer AJ, van den Heuvel WJ. The wheelchair circuit: reliability of a test to assess mobility in persons with spinal cord injuries. Arch Phys Med Rehabil. 2002;83:1783-1788.

22. Sol M, De Groot JF, Verschuren O, Visser-Meily A. Effectiveness of wheelchair skills training in children using a manual wheelchair. In: Proceedings of the World Confederation for Physical Therapy Congress 2017; July 2-4, 2017; Cape Town, South Africa. Abstract RR-PO-02-12-TUE.

23. Portney LG, Watkins MP. Foundations of Clinical Research: Application to Practice. 3rd ed. Upper Saddle River, NJ: Prentice Hall; 2009.

24. Cowan RE, Nash MS, de Groot S, van der Woude LH. Adapted manual wheelchair circuit: testretest reliability and discriminative validity in persons with spinal cord injury. Arch Phys Med Rehabil. 2011;92:1270-1280.

25. Fliess-Douer O, Van Der Woude LH, Vanlandewijck YC. Reliability of the test of wheeled mobility (TOWM) and the short Wheelie test. Arch Phys Med Rehabil. 2013;94:761-770.

26. Sawatzky B, Hers N, MacGillivray MK. Relationships between wheeling parameters and wheelchair skills in adults and children with SCI. Spinal Cord. 2015;53:561-564.

27. Kilkens OJ, Dallmeijer AJ, Nene AV, Post MW, van der Woude LH. The longitudinal relation between physical capacity and wheelchair skill performance during inpatient rehabilitation of people with spinal cord injury. Arch Phys Med Rehabil. 2005;86:1575- 1581.

\section{Tables and Figures}

Figure. Distribution of baseline Utrecht Pediatric Wheelchair Mobility Skills Test (UP-WMST) 2.0 scores in the total sample.

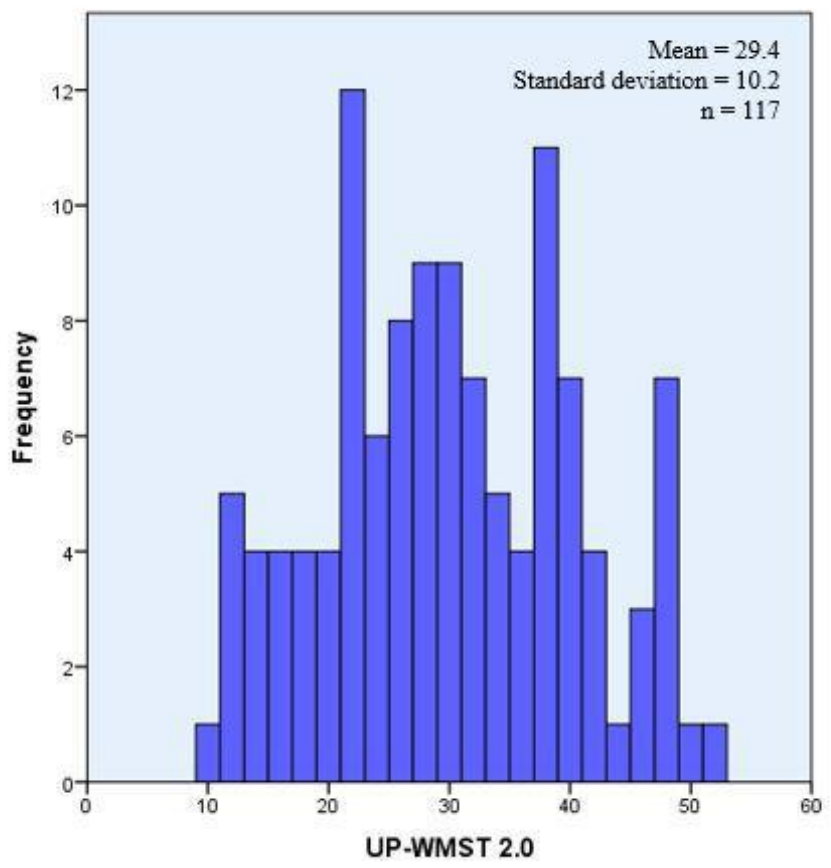


Sol, M.E., Groot, J.F. de, Zwinkels, M., Kruitwagen, C.L., Vershuren, O. Utrecht pediatric wheelchair mobility skills test: reliability, validity, and responsiveness in youths using a manual wheelchair.

Physical Therapy: 2019, 8, 1098-1106

Table 1. Distribution of Time Scores Per Item and Determination of Cutoff Times for the UP-WMST $2.0^{a}$

\begin{tabular}{|c|c|c|c|c|c|c|c|c|c|}
\hline \multirow[t]{2}{*}{ UP-WMST Item List } & \multirow{2}{*}{$\begin{array}{l}\text { No. of } \\
\text { Completed } \\
\text { Items }\end{array}$} & \multirow[t]{2}{*}{ Mean } & \multirow[t]{2}{*}{ SD } & \multirow{2}{*}{$\begin{array}{c}\text { 25th } \\
\text { Percentile }\end{array}$} & \multirow{2}{*}{$\begin{array}{c}\text { 75th } \\
\text { Percentile }\end{array}$} & \multicolumn{4}{|c|}{ Cutoff Time at Rank of: } \\
\hline & & & & & & 0 & 1 & 2 & 3 \\
\hline 1. Propulsion forward (s) & 117 & 6.92 & 3.04 & 5.14 & 7.45 & NC & $>7.5$ & $5-7.5$ & $<5$ \\
\hline 2. Propulsion backward (s) & 113 & 8.23 & 5.23 & 5.37 & 9.15 & $\mathrm{NC}$ & $>9$ & $5.5-9$ & $<5.5$ \\
\hline 3. Rolls on soft surface (s) & 115 & 2.98 & 1.57 & 2.11 & 3.31 & $\mathrm{NC}$ & $>3.5$ & $2-3.5$ & $<2$ \\
\hline $\begin{array}{l}\text { 4. Turns } 90^{\circ} \text { while moving } \\
\text { forward }(\mathrm{s})\end{array}$ & 117 & 5,07 & 2.22 & 3.85 & 5.49 & $\mathrm{NC}$ & $>5.5$ & $4-5.5$ & $<4$ \\
\hline $\begin{array}{l}\text { 5. Turns } 90^{\circ} \text { while moving } \\
\text { backward (s) }\end{array}$ & 115 & 9,35 & 7.61 & 5.34 & 10.02 & $\mathrm{NC}$ & $>10$ & $5.5-10$ & $<5.5$ \\
\hline 6. Turns $180^{\circ}$ in place $(\mathrm{s})$ & 114 & 4.00 & 3.20 & 2.34 & 4.56 & NC & $>4.5$ & $2.5-4.5$ & $<2.5$ \\
\hline 7. Sudden stop (s) & 115 & 1.57 & 5.14 & 0.78 & 1.34 & $\mathrm{NC}$ & $>1.5$ & $1-1.5$ & $<1$ \\
\hline \multicolumn{10}{|l|}{ 8. Opening/closing a door (s) } \\
\hline Away & 114 & 12.56 & 7.43 & 6.83 & 17.43 & $\mathrm{NC}$ & $>17.5$ & $7-17.5$ & $<7$ \\
\hline Toward & 98 & 18.37 & 12.69 & 10.13 & 22.54 & NC & $>22.5$ & $10-22.5$ & $<10$ \\
\hline 9. Figure-of-eight shape (s) & 115 & 11.48 & 6.18 & 8.41 & 12.18 & NC & $>12$ & $8.5-12$ & $<8.5$ \\
\hline 10. Holding a wheelie (s) & 69 & 11.67 & 13.51 & 0.79 & 30.00 & NC & $<2$ & $2-30$ & $>30$ \\
\hline 11. Slope ascent (s) & 83 & 4.97 & 2.39 & 3.48 & 6.03 & NC & $>6$ & $3.5-6$ & $<3.5$ \\
\hline 12. Slope descent (s) & 117 & 3.12 & 2.92 & 1.88 & 3.12 & NC & $>3$ & $2-3$ & $<2$ \\
\hline \multicolumn{10}{|l|}{ 13. Platform ascending (s) } \\
\hline $5 \mathrm{~cm}$ & 92 & 5.58 & 4.42 & 3.03 & 6.55 & $\mathrm{NC}$ & $>6.5$ & $3-6.5$ & $<3$ \\
\hline $10 \mathrm{~cm}$ & 25 & 4.79 & 3.65 & 2.85 & 4.46 & $\mathrm{NC}$ & $>4.5$ & $3-4.5$ & $<3$ \\
\hline \multicolumn{10}{|l|}{ 14. Platform descending (s) } \\
\hline $5 \mathrm{~cm}$ & 116 & 2.34 & 1.64 & 1.50 & 2.33 & NC & $>2.5$ & $1.5-2.5$ & $<1.5$ \\
\hline $10 \mathrm{~cm}$ & 93 & 3.68 & 2.52 & 2.08 & 4.32 & $\mathrm{NC}$ & $>4.5$ & $2-4.5$ & $<2$ \\
\hline 15. Doorstep (s) & 113 & 3.85 & 5.09 & 2.18 & 3.87 & $\mathrm{NC}$ & $>4$ & $2-4$ & $<2$ \\
\hline
\end{tabular}

${ }^{a} \mathrm{NC}=$ not completed; UP-WMST $=$ Utrecht Pediatric Wheelchair Mobility Skills Test.

Table 2.

Ordinal Scale of the UP-WMST $2.0^{\circ}$

\begin{tabular}{|c|l|}
\hline Rank & Description \\
\hline 0 & $\begin{array}{l}\text { Not able to complete item or time to task } \\
\text { completion }>60 \mathrm{~s}\end{array}$ \\
\hline 1 & $>75$ th percentile \\
\hline 2 & Between 25 th and 75 th percentiles \\
\hline 3 & $<25$ th percentile \\
\hline
\end{tabular}

${ }^{a}$ UP-WMST $=$ Utrecht Pediatric Wheelchair Mobility Skills Test. 
Sol, M.E., Groot, J.F. de, Zwinkels, M., Kruitwagen, C.L., Vershuren, O. Utrecht pediatric wheelchair mobility skills test: reliability, validity, and responsiveness in youths using a manual wheelchair. Physical Therapy: 2019, 8, 1098-1106

Table 3.

Participant Characteristics and UP-WMST 2.0 Scores $^{a}$

\begin{tabular}{|l|c|c|c|}
\hline Parameter & $\begin{array}{c}\text { Reliability } \\
\text { Study }\end{array}$ & Validity Study & $\begin{array}{c}\text { Responsiveness } \\
\text { (Pilot) Study }\end{array}$ \\
\hline No. of participants & & & 23 \\
\hline Total & 30 & 117 & 21 \\
\hline Children & 12 & 56 & 2 \\
\hline Adolescents & 18 & 61 & $9.3(1.9)$ \\
\hline Age, $y$, mean (SD) & $12.5(3.4)$ & $12.4(3.4)$ & 16 \\
\hline Sex (no. of participants) & & & 7 \\
\hline Male & 18 & 71 & 11 \\
\hline Female & 12 & 46 & 5 \\
\hline Diagnoses (no. of participants) & & & 1 \\
\hline Spina bifida & 15 & 31 & 6 \\
\hline Cerebral palsy & 13 & 47 & \\
\hline Muscle disorder & 1 & 11 & $29.2(9.0)$ \\
\hline Other & 1 & 28 & \\
\hline UP-WMST 2.0 score, mean (SD) & & & $38.9(8.8)$ \\
\hline Baseline & $29.4(11.0)$ & $29.4(10.2)$ & \\
\hline Retest (n=29) & $31.3(10.4)$ & & \\
\hline After training (n = 20) & & & \\
\hline
\end{tabular}

${ }^{a}$ UP-WMST $=$ Utrecht Pediatric Wheelchair Mobility Skills Test. 
Sol, M.E., Groot, J.F. de, Zwinkels, M., Kruitwagen, C.L., Vershuren, O. Utrecht pediatric wheelchair mobility skills test: reliability, validity, and responsiveness in youths using a manual wheelchair. Physical Therapy: 2019, 8, 1098-1106

Table 4.

Descriptive Statistics for UP-WMST 2.0 Items, Subitems and Total Score ${ }^{a}$

\begin{tabular}{|c|c|c|c|c|c|c|c|c|c|c|c|c|}
\hline \multirow[t]{2}{*}{ Item } & \multicolumn{5}{|c|}{ Test } & \multicolumn{5}{|c|}{ Retest } & \multicolumn{2}{|c|}{ Reliability } \\
\hline & $\mathrm{n}^{b}$ & Median & Mean & Q1-Q3 & SD & $\mathbf{n}^{b}$ & Median & Mean & Q1-Q3 & SD & $\begin{array}{c}\text { Weighted } \\
\text { Cohen Kappa } \\
\text { Coefficient }\end{array}$ & $I^{\prime C} C_{\text {agreement }}$ \\
\hline 1. Propulsion forward & 29 & 2 & & $\begin{array}{c}2.00- \\
3.00\end{array}$ & & 29 & 2 & & $2.00-2.00$ & & 0.80 & \\
\hline 2. Propulsion backward & 29 & 2 & & $\begin{array}{l}1.00- \\
3.00\end{array}$ & & 29 & 2 & & $1.50-3.00$ & & 0.63 & \\
\hline 3. Rolls on soft surface & 29 & 2 & & $\begin{array}{l}1.00- \\
2.00\end{array}$ & & 29 & 2 & & $1.50-2.00$ & & 0.71 & \\
\hline $\begin{array}{l}\text { 4. Turns } 90^{\circ} \text { while moving } \\
\text { forward }\end{array}$ & 29 & 2 & & $\begin{array}{l}1.50- \\
3.00\end{array}$ & & 29 & 2 & & $1.00-3.00$ & & 0.78 & \\
\hline $\begin{array}{l}\text { 5. Turns } 90^{\circ} \text { while moving } \\
\text { backward }\end{array}$ & 29 & 2 & & $\begin{array}{l}1.00- \\
2.50\end{array}$ & & 29 & 2 & & $1.00-3.00$ & & 0.77 & \\
\hline 6. Turns $180^{\circ}$ in place & 29 & 2 & & $\begin{array}{l}1.00- \\
2.50\end{array}$ & & 29 & 2 & & $2.00-2.50$ & & 0.63 & \\
\hline 7. Sudden stop & 29 & 2 & & $\begin{array}{l}2.00- \\
3.00\end{array}$ & & 29 & 2 & & $2.00-3.00$ & & 0.50 & \\
\hline \multicolumn{13}{|l|}{ 8. Opening/closing a door } \\
\hline Away & 28 & 2 & & $\begin{array}{c}2.00- \\
3.00\end{array}$ & & 29 & 2 & & $2.00-3.00$ & & 0.67 & \\
\hline Toward & 29 & 2 & & $\begin{array}{l}1.25- \\
3.00\end{array}$ & & 27 & 2 & & $2.00-3.00$ & & 0.88 & \\
\hline 9. Figure-of-eight shape & 29 & 2 & & $\begin{array}{c}2.00- \\
3.00\end{array}$ & & 29 & 2 & & $1.00-3.00$ & & 0.74 & \\
\hline 10. Holding a wheelie & 28 & 1 & & $\begin{array}{l}0.00- \\
2.00\end{array}$ & & 27 & 1 & & $1.00-3.00$ & & 0.80 & \\
\hline
\end{tabular}

\begin{tabular}{|l|c|c|c|c|c|c|c|c|c|c|c|}
\hline 11. Slope ascent & 29 & 1 & & $\begin{array}{c}0.00- \\
3.00\end{array}$ & & 29 & 2 & & $0.00-3.00$ & 0.89 & \\
\hline 12. Slope descent & 29 & 2 & & $\begin{array}{c}1.00- \\
3.00\end{array}$ & & 29 & 2 & & $1.00-3.00$ & 0.67 & \\
\hline 13. Platform ascending & & & & & & & & & & & \\
\hline $5 \mathrm{~cm}$ & 29 & 2 & & $\begin{array}{c}0.50- \\
2.00\end{array}$ & & 29 & 2 & & $1.00-3.00$ & & 0.88 \\
\hline $10 \mathrm{~cm}$ & 28 & 0 & & $\begin{array}{c}0.00- \\
0.75\end{array}$ & 29 & 0 & & $0.00-0.50$ & & 0.98 \\
\hline 14. Platform descending & & & & & & & & & & & \\
\hline $5 \mathrm{~cm}$ & 29 & 2 & & $\begin{array}{c}1.00- \\
2.00\end{array}$ & & 29 & 2 & & $1.50-2.50$ & & 0.79 \\
\hline $10 \mathrm{~cm}$ & 27 & 2 & & $\begin{array}{c}1.00- \\
2.00\end{array}$ & & 29 & 2 & & $1.00-2.50$ & & 0.74 \\
\hline 15. Doorstep & 29 & 2 & & $\begin{array}{c}2.00- \\
2.00\end{array}$ & & 29 & 2 & & $1.00-2.00$ & & 0.69 \\
\hline Total UP-WMST 2.0 score & 29 & & 30.0 & & 10.7 & 29 & & 31.3 & & 10.4 & \\
\hline
\end{tabular}

${ }^{a} \mathrm{ICC}_{\text {agreement }}=$ Intraclass correlation coefficient; $\mathrm{Q} 1=$ first quartile; $\mathrm{Q} 3=$ third quartile; UP-WMST = Utrecht Pediatric Wheelchair Mobility Skills Test.

${ }^{b} \mathrm{n}=$ Number of participants. 
Sol, M.E., Groot, J.F. de, Zwinkels, M., Kruitwagen, C.L., Vershuren, O. Utrecht pediatric wheelchair mobility skills test: reliability, validity, and responsiveness in youths using a manual wheelchair. Physical Therapy: 2019, 8, 1098-1106

\section{Table 5.}

Comparison of Total UP-WMST 2.0 Scores Between and Within Groups ${ }^{a}$

\begin{tabular}{|l|c|c|c|c|c|}
\hline Parameter & $\begin{array}{c}\text { No. of } \\
\text { Participants }\end{array}$ & Mean & SD & Range & $\boldsymbol{P}$ \\
\hline Age & & & & & \\
\hline Children & 56 & 26.6 & 9.3 & $10-47$ & \\
\hline Adolescents & 61 & 32.0 & 10.5 & $11-51$ & $<.01^{D}$ \\
\hline Diagnosis & & & & & \\
\hline Cerebral palsy & 47 & 26.1 & 9.4 & $11-48$ & \\
\hline Spina bifida & 31 & 35.2 & 9 & $13-48$ & $<.001^{b}$ \\
\hline Responsiveness & & & & & \\
\hline Before training & 20 & 30.6 & 8.8 & $14-47$ & \\
\hline After training & 20 & 38.9 & 8.8 & $26-50$ & $<.001^{C}$ \\
\hline
\end{tabular}

${ }^{a}$ UP-WMST $=$ Utrecht Pediatric Wheelchair Mobility Skills Test.

${ }^{b}$ From independent-sample $t$ test.

${ }^{c}$ From paired-sample $t$ test. 